\title{
Design and realization of the emergency unit of video frequency switch matrix
}

\author{
Feng Yue, Yu Wei
}

China Satellite Maritime Tracking and Control Department, Jiangyin, 214431, China

Keywords: USB; UCB; video switch matrix; MC3486

\begin{abstract}
In order to meet the lunar exploration project three follow-up mission requirements, ship-borne unified monitoring and control system for adaptive transformation, which video switch matrix as the input and output interface is not exactly the same, if the goal of horizon fault, can only replace the backup plug Box method, time-consuming effort, the task risk is too large. This paper designs a set of unified system video switch matrix emergency switch unit, which can realize the emergency signal processing of the unified system to track the link. It can effectively improve the emergency handling capability of the tracking system and reduce the task risk.
\end{abstract}

\section{Introduction}

Unified monitoring and control system mainly refers to the unified S-band measurement and control system (referred to as USB) and unified C-band measurement and control system (UCB), to meet the lunar exploration project three follow-up tasks, Tracking measurement and high rate FM telemetry tracking and receiving functions. After the transformation of the baseband to meet the tracking function, the video switch matrix interface also changed accordingly, to meet the original USB tracking receiver, the original UCB tracking receiver, USB baseband tracking signal input and output. At present, for the baseband subsystem, the tracking power points and video switching matrix in the unified system tracking link are single-point failure. If the equipment fails in the task, the tracking power sub-box can be shorted by the cable head., And the video switch matrix as the input and output interfaces are not exactly the same, can not be directly short even if the target level out of the video switch matrix after the failure can only be used to replace the backup subbox method, but the replacement of time and effort, Too large. This paper designs a set of unified system video switch matrix emergency switch unit, which can realize the emergency signal processing of the unified system to track the link. It can effectively improve the emergency handling capability of the tracking system and reduce the task risk.

\section{Feasibility Analysis}

\section{Module structure and function analysis.}

The basic components of the ship-borne unified monitoring and control system after the adaptability of the same, still by the days of feeding sub-system, transmitting sub-systems, high-frequency receiving sub-system, multi-functional digital baseband subsystem, digital tracking receiver, monitoring sub-system, System, time-frequency system, test standard calibration system, low-light television subsystems and other components. Equipment transformation specific content includes multi-functional baseband sub-system transformation of four baseband, the new research a video switch matrix. The new video switching matrix is mainly composed of analog module, reference forwarding module, general control module and linear power supply. The video switching matrix receives the tracking lock indication signal, locking voltage and angle error sent by the three tracking devices simultaneously. Voltage and according to the system settings to select a group of signals sent to the servo system for automatic tracking, on the other hand to receive the $25 \mathrm{~Hz}$ matching box sent to the same direction and quadrature reference signal to the USB tracking receiver separately. From the tracking principle we can see, $25 \mathrm{~Hz}$ with the orthogonal reference signal is the USB system to guide the antenna's conical scan from the tracking method to demodulate the azimuth angle of the azimuth error voltage to provide the basis for the guidance 
antenna only in the main antenna to find the target before the reference in the actual The effect can be ignored in the tracking project. Therefore, the video switch matrix emergency switching unit in the design only needs to consider the main antenna tracking lock indication, locking voltage and angular error voltage transmission.

\section{Tracking signal transmission analysis.}

New research video switch matrix rear panel device structure shown in Figure 1, the left half of the main AGC lock indication and voltage input and output, the new research baseband angle error signal input; the right half of the main USB / UCB original tracking receiver angle Error signal input and video switching matrix angular error output. If it works in USB mode, the corresponding USB tracking receiver AGC voltage and lock indication input in X9 should be short-circuited with the corresponding USB tracking receiver AGC voltage and AGC lock indication output in X10. X103 shorted, X301 and X303 shorted; Similarly, if the work in UCB mode, the emergency need to X9 (aviation head 37 pin) in the corresponding UCB tracking receiver AGC voltage and AGC lock instruction input and X10 19 pin) corresponding to the UCB tracking receiver AGC voltage and AGC lock instruction output shorted, the X501 and X503 angle error part of the shorted, X701 and X703 shorted; if the work in the transformation of the baseband tracking mode, the emergency needs The corresponding azimuth error voltage and pitch error voltage of X15 / X16 / X17 / X18 (aviation head 10 pins) are shorted to X103, X303 is shorted, AGC voltage and AGC lock indication input are the same as X10 (aviation head 19 pin) The AGC voltage and AGC lock indication output are shorted. As these signals are analog, can be directly shorted physical to achieve emergency response.

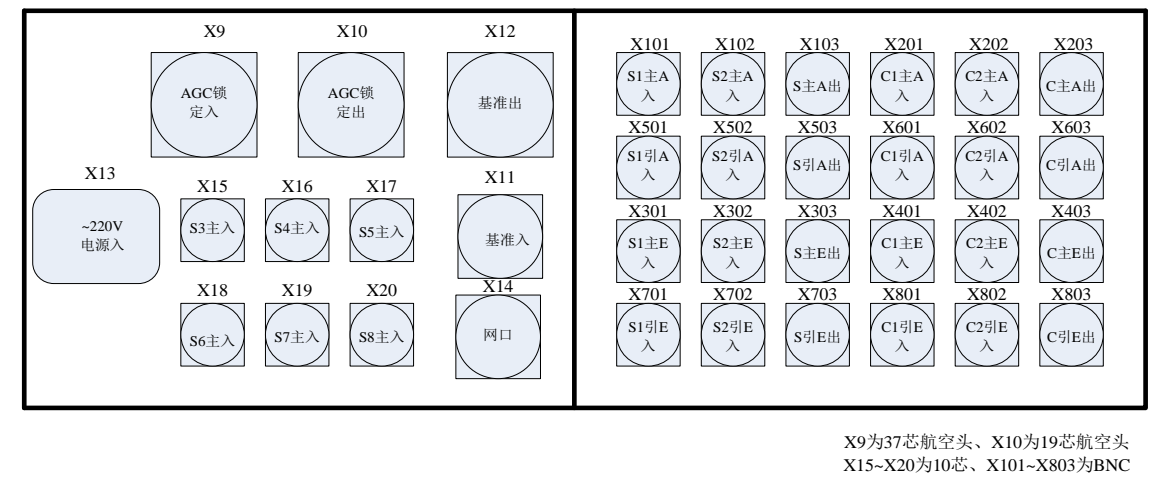

Figure 1. New research video switch matrix rear panel device structure

X9 / X16 / X17 / X18 are 19-pin air connector, X9 interface pin definition in Table 1, X10 interface pin definition in Table 2, X15 / X16 / X17 / X18 interface pin definition in Table 3.

Table 1. Definition of the X9 video switching matrix X9 interface

\begin{tabular}{|c|c|c|c|c|}
\hline Source & $\begin{array}{c}\text { Pin } \\
\text { number }\end{array}$ & Signal content & $\begin{array}{c}\text { Circuit diagram } \\
\text { Destination }\end{array}$ & $\begin{array}{c}\text { Circuit Diagram Pin } \\
\text { Number }\end{array}$ \\
\hline X9 & 1 & S1-AGC & J1 & 1 \\
\hline X9 & 3 & S2-AGC & - & 2 \\
\hline X9 & 5 & S1-LOCK & J1 & - \\
\hline X9 & 8 & S2-LOCK & - & - \\
\hline X9 & 23 & C1-AGC & J1 & 4 \\
\hline X9 & 27 & C2-AGC & - & - \\
\hline X9 & 29 & C1-LOCK & J1 & $6,7,8,9$ \\
\hline X9 & 32 & C2-LOCK & - & J1 \\
\hline X9 & 10,11 & GND & & \\
\hline
\end{tabular}


Table 2. Definition of X10 video switch matrix X10 interface

\begin{tabular}{|c|c|c|c|c|}
\hline Source & $\begin{array}{c}\text { Pin } \\
\text { number }\end{array}$ & Signal content & $\begin{array}{c}\text { Circuit diagram } \\
\text { Destination }\end{array}$ & $\begin{array}{c}\text { Circuit Diagram } \\
\text { Pin Number }\end{array}$ \\
\hline $\mathrm{X} 10$ & 1 & S-AGC & J2 & 2 \\
\hline $\mathrm{X} 10$ & 6 & S-LOCK+ & J2 & 1 \\
\hline $\mathrm{X} 10$ & 7 & S-LOCK- & J2 & 6 \\
\hline $\mathrm{X} 10$ & 3 & C-AGC & J2 & 3 \\
\hline $\mathrm{X} 10$ & 8 & C-LOCK+ & J2 & 8 \\
\hline $\mathrm{X} 10$ & 9 & C-LOCK- & J2 & 7,9 \\
\hline $\mathrm{X} 10$ & 10,11 & GND & J2 & \\
\hline
\end{tabular}

Table 3. New research video switching matrix X15 / X16 / X17 / X18 interface definition

\begin{tabular}{|c|c|c|c|c|}
\hline Source & $\begin{array}{c}\text { Pin } \\
\text { number }\end{array}$ & $\begin{array}{c}\text { Signal } \\
\text { content }\end{array}$ & $\begin{array}{c}\text { Circuit diagram } \\
\text { Destination }\end{array}$ & $\begin{array}{c}\text { Circuit Diagram Pin } \\
\text { Number }\end{array}$ \\
\hline $\mathrm{X} 15 / \mathrm{X} 16 / \mathrm{X} 17 / \mathrm{X} 18$ & 1 & S3-A & J3 & 1 \\
\hline $\mathrm{X} 15 / \mathrm{X} 16 / \mathrm{X} 17 / \mathrm{X} 18$ & 3 & S3-E & J3 & 2 \\
\hline $\mathrm{X} 15 / \mathrm{X} 16 / \mathrm{X} 17 / \mathrm{X} 18$ & 5 & S3-LOCK & $\mathrm{J} 3$ & 3 \\
\hline $\mathrm{X} 15 / \mathrm{X} 16 / \mathrm{X} 17 / \mathrm{X} 18$ & 7 & S3-AGC & $\mathrm{J} 3$ & 4 \\
\hline $\mathrm{X} 15 / \mathrm{X} 16 / \mathrm{X} 17 / \mathrm{X} 18$ & $2,4,6,8$ & GND & $\mathrm{J} 3$ & $6,7,8,9$ \\
\hline
\end{tabular}

\section{Lock indication signal transmission analysis.}

Servo system of the new research video switch matrix sent out of the lock instruction signal processing circuit shown in Figure 2, which lock decision MC3486 chip, MC3486 chip logic function table and chip pin definition diagram shown in Figure 3.

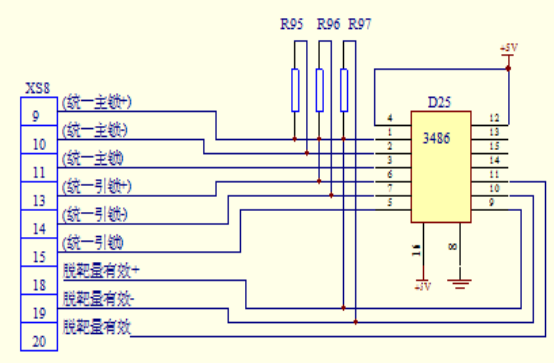

Figure 2. Differential signal processing circuit diagram
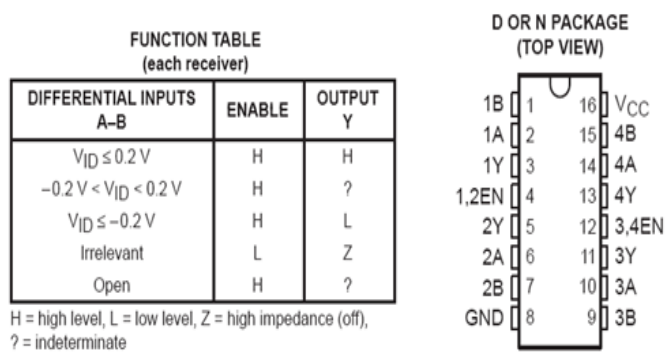

Figure 3. MC3486 chip logic function table and chip pin definition diagram

The servo system determines the locked output state by the difference of the two locking signals. If the positive and negative difference is more than or equal to $0.2 \mathrm{~V}$, the output will be high, indicating that the tracking lock is valid. If the difference is less than or equal to $-0.2 \mathrm{~V}$, the output is low Level, that is, the device is in the lockout state; if the difference between the two, the lock state is uncertain.

Actual measurements show that when the USB tracking receiver or the new research baseband locked carrier, X9 aviation head 5 input high level 4.99V, this time, X10 aviation head 6, 7 output voltage respectively $4.65 \mathrm{~V}$ and $0 \mathrm{~V}$; When the USB tracking receiver or new baseband loss of lock, $\mathrm{X} 9$ aviation head 5 input low $0.01 \mathrm{~V}$, this time, X10 aviation head 6,7 output voltage respectively $0 \mathrm{~V}, 4.65 \mathrm{~V}$. The level difference between the positive and negative interfaces satisfies the MC3486 
chip's lock-in decision condition, as well as the output voltage in the UCB mode.

\section{Design of Video Switch Matrix Emergency Switch Unit}

To sum up, in the video switch matrix emergency switch unit design not only to meet the relevant analog voltage shorted physical layer requirements, but also pay attention to meet the lock instruction signal differential output requirements, the corresponding design circuit shown in Figure 4.

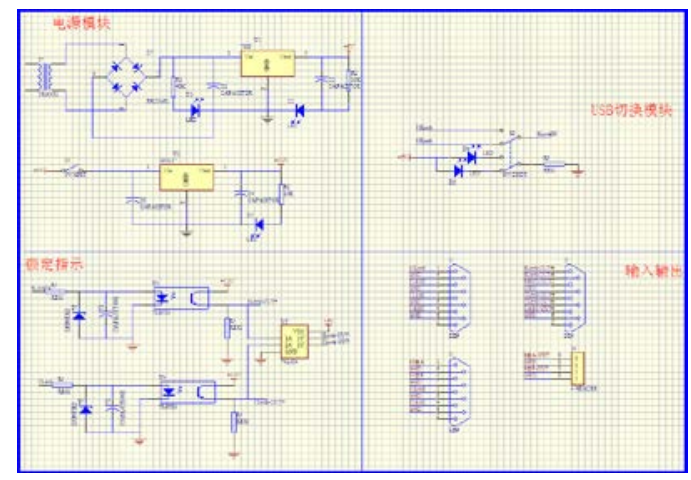

Figure 4. Video Switch Matrix Emergency Switch Unit Circuit Diagram

Power supply module using two integrated circuits 7905 and SP1117, respectively, to achieve 5V, $3.3 \mathrm{~V}$ voltage output; USB switch module to achieve the original USB tracking receiver and transform the baseband input selection; input and output modules to transform the baseband angle error analog signal input and output ; Locking instruction module mainly uses two photosensitive devices TLP521 will input the lock instruction signal into a stable analog voltage, and through the 74LS04 chip to complete the lock instruction voltage differential output. The whole system will achieve the video switch matrix emergency signal switching and output.

\section{Summary}

It is proved that the video switch matrix emergency switch unit can be connected to the system through the simulation of the original video switch matrix fault, which can effectively transmit the tracking lock indication and angle error signal. The whole system is stable and has achieved the expected effect. The proposed video switching matrix emergency switching unit can effectively reduce the original video switch matrix as a single point of failure of the hazard factor, the replacement of the previous backup video switch matrix time-consuming and labor-intensive weaknesses have greatly improved, effectively guarantee the emergency situation USB / UCB tracking signal accurate transmission, the application of shipborne monitoring and control equipment in the actual work, has undergone a number of actual combat tests for the successful completion of space missions to provide a strong guarantee.

\section{Reference}

[1] Elliott Kaplan, Christopher Hegarty. Understanding GPS theory and Application, Second Edition [M]. Boston: Aztec House, 2014

[2] James B.Y. Tsui. Fundamentals of Global Positioning System Receivers, 2ED [M]. John Wiley \& Sons. Inc, 2011

[3] J.K Holmes, C Chen. Acquisition time performance of PN spread-spectrum systems[J]. IEEE Transactions on communications,2011:778-784 